University of South Carolina

Scholar Commons

3-1977

\title{
A Random Differential-Equation Approach to Probability Distribution of BOD and DO in Streams
}

\author{
William J. Padgett \\ University of South Carolina - Columbia, padgettw@bellsouth.net \\ G Schultz \\ Chris P. Tsokos
}

Follow this and additional works at: https://scholarcommons.sc.edu/math_facpub

Part of the Mathematics Commons, and the Statistics and Probability Commons

\section{Publication Info}

Siam Journal on Applied Mathematics, Volume 32, Issue 2, 1977, pages 467-483.

(c) 1997 by Society for Industrial and Applied Mathematics

This Article is brought to you by the Mathematics, Department of at Scholar Commons. It has been accepted for inclusion in Faculty Publications by an authorized administrator of Scholar Commons. For more information, please contact digres@mailbox.sc.edu. 


\title{
A RANDOM DIFFERENTIAL EQUATION APPROACH TO THE PROBABILITY DISTRIBUTION OF BOD AND DO IN STREAMS*
}

\author{
W. J. PADGETT, $\uparrow$ G. SCHULTZ $\ddagger$ AND CHRIS P. TSOKOS§
}

\begin{abstract}
In this paper a stochastic model for stream pollution is given which involves a random differential equation of the form

$$
\dot{\mathbf{X}}(t)=\mathbf{A X}(t)+\mathbf{Y}, \quad t \geqq 0,
$$

where $\mathbf{X}(t)$ is a two-dimensional vector-valued stochastic process with the first component giving the biochemical oxygen demand (BOD) and the second component representing the dissolved oxygen (DO) at distance $t$ downstream from the source of pollution. The fundamental Liouville's theorem is utilized to obtain the probability distribution of the solution of $\left({ }^{*}\right), \mathbf{X}(t)$, at each $t$ with various distributional assumptions on the random initial conditions and random inhomogeneous term. Computer simulations of the trajectories of the BOD and DO processes as well as the mean and variance functions are given for several initial distributions and are compared with the deterministic results.
\end{abstract}

1. Introduction. Recently, it has become increasingly evident that the world's most valuable natural resources-air and water-are being endangered by the activities of civilized man. The water supply is being endangered by the disposal of organic (and other) waste materials into natural bodies of water by municipalities and industries. This pollution has become a major concern of the scientific community, and various regulatory agencies have specified minimum levels for dissolved oxygen (DO) in lakes and streams. These minimum levels of DO are extremely important since if DO falls below a certain threshold value, the fish and other living organisms in the body of water may die.

Many organic compounds discharged into lakes and streams are effectively degraded into inoffensive components by the action of bacteria in the water. As the organisms in a body of water degrade the pollution, they require oxygen, and hence use the dissolved oxygen (DO) in the water. Oxygen-consuming pollutants are measured in terms of the amount of oxygen required by the bacteria to stabilize them, called the biochemical oxygen demand (BOD). It is generally accepted that the BOD and DO, measured in parts per million (ppm), are the primary indicators of water quality in a stream or river. Dissolved oxygen is obtained directly from the air by aeration and indirectly through the photosynthetic process of aquatic plants.

Beginning with the classical equations of Streeter and Phelps [9], various mathematical models have been proposed for describing the behavior of the DO and BOD profiles along a stretch of natural stream [1]-[2], [4]-[7], [10]. Dobbins [1] obtained a pair of differential equations whose solution described the BOD and DO at downstream points from a pollution source. Thayer and Krutchkoff [10] used a generating function technique to obtain the approximate (discrete)

* Received by the editors February 25, 1976.

$\dagger$ Department of Mathematics and Computer Science, University of South Carolina, Columbia, South Carolina 29208.

‡ Science Division, St. Petersburg Jr. College, Clearwater Campus, Clearwater, Florida 33515.

$\S$ Department of Mathematics, University of South Florida, Tampa, Florida 33620. Supported by the U.S. Air Force Office of Scientific Research under Grant AFOSR-75-2711. 
probability distributions for DO and BOD at each distance $t$ downstream from the source of pollution. Also, Loucks and Lynn [4] used methods of Markov chains to predict the probability distribution of minimum DO levels. Both of these stochastic models assumed that DO or BOD concentration may be in only one of a finite number of possible states at any given time. More recently, Padgett [7] used the theory of random differential equations to obtain a model for stream pollution. The purpose of the present paper is to extend the results of Padgett [7] in that Liouville's theorem is utilized to obtain the joint probability distribution of the BOD and DO processes at each distance $t$ downstream from a point source of pollution. The importance of this result is that the probability distribution may be used to obtain probability statements about the DO levels or to obtain the mean and variance of DO and BOD at each distance $t$ downstream from the major source of pollution. Hence, these results are important in determining the possibilities of fish kills in the stream. Also, Padgett [7] assumed that the random variables involved were independent, whereas in this paper they may be assumed to be correlated.

The general assumptions that will be made here are those made by Thayer and Krutchkoff [10] and by Padgett [7]. Thus, it will be assumed that there are five major activities in the stream: (i) The pollution (BOD) and DO are decreased by the action of bacteria. The rate of decrease is assumed to be proportional to the amount of pollution present with proportionality constant $k_{1}$ in units of dissolved oxygen per day (ppm), and there is always some oxygen present. (ii) The dissolved oxygen is increased due to reaeration at a rate proportional to the dissolved oxygen deficit (which is the DO saturation concentration minus the actual DO concentration) with proportionality constant $k_{2}$ in units of dissolved oxygen per day (ppm). (iii) The pollution only is decreased by sedimentation and adsorption at a rate proportional to the amount of pollution present with proportionality constant $k_{3}$ in units of dissolved oxygen per day (ppm). (iv) The pollution is increased from small sources along the stretch of stream with rate $l_{a}$ in ppm per day which is independent of the amount of pollution present. (v) The dissolved oxygen is decreased at a rate $d_{B}$ in ppm per day. The variable $d_{B}$ may have positive or negative values and represents the net change in dissolved oxygen due to the Benthal demand and respiration and photosynthesis of plants.

In $\S 2$ of this paper we will present the stochastic model in terms of random differential equations and discuss the solution processes. In $\S 3$, Liouville's theorem will be applied to obtain the jeint probability distribution of the solutions to the random differential equations. Examples and computer simulations will be given in $\S 4$, indicating how the results of $\S 3$ may be utilized in studying the statistical properties of the BOD and DO processes.

2. The random differential equations and their solution. Assuming, in addition to the assumptions (i)-(v) in $\S 1$, that the stream flow was steady and uniform and that the conditions at every cross section were unchanged with time, Dobbins [1] obtained the deterministic differential equations for BOD and DO given by

$$
\begin{aligned}
& -u \dot{l}(t)-\left(k_{1}+k_{3}\right) l(t)+l_{a}=0, \\
& -u \dot{c}(t)+k_{2}\left[c_{S}-c(t)\right]-k_{1} l(t)-d_{B}=0,
\end{aligned}
$$


where the dot denotes the derivative with respect to $t, t$ is the distance downstream from the pollution source, $l(t)$ is the (first stage) BOD in ppm at distance $t, u$ is the average velocity along the stretch, $c_{S}$ is the saturation concentration for dissolved oxygen, and $c(t)$ is the concentration of $\mathrm{DO}$ (in ppm) at distance $t$ downstream. The initial conditions for the system $(2.1)$ were $l(0)=l_{0}$ and $c(0)=c_{0}$. Also, the longitudinal dispersion effect in the stream was negligible as shown in [1] and hence does not appear in (2.1). The assumptions (i)-(v) of $\S 1$ concerning the biological processes in the stream were made to simplify the complicated mathematical equations that necessarily would arise when a complex biological system is being modeled. However, as in the formulation of most mathematical models, the assumptions seem to be reasonable and realistic and do yield the workable model (2.1).

Padgett [7] pointed out that the quantities $k_{1}, k_{2}, k_{3}, u, l_{a}, c_{S}, d_{B}, l_{0}$ and $c_{0}$ are more realistically considered to be random variables which have certain probability distributions rather than as physical constants as they had been treated by Thayer and Krutchkoff [10] and Loucks and Lynn [4]. This means that some of the uncertainty in the modeling of the BOD and DO processes may be expressed in the form of randomness in certain of the variables involved in the model. Therefore, in [7] the system of random differential equations given by

$$
\begin{aligned}
\dot{\mathbf{X}}(t) & =\mathbf{A X}(t)+\mathbf{Y}, \quad t \geqq 0 \quad\left(\cdot=\frac{d}{d t}\right), \\
\mathbf{X}(t) & =\left(\begin{array}{l}
L(t) \\
C(t)
\end{array}\right), \quad \mathbf{Y}=\left(\begin{array}{c}
L_{a} / u \\
\left(k_{2} c_{S}-D_{B}\right) / u
\end{array}\right), \\
\mathbf{A} & =\left(\begin{array}{cc}
-\left(k_{1}+k_{3}\right) / u & 0 \\
-k_{1} / u & -k_{2} / u
\end{array}\right),
\end{aligned}
$$

and

$$
\mathbf{X}(0)=\mathbf{X}_{0}=\left(\begin{array}{l}
L_{0} \\
C_{0}
\end{array}\right)
$$

was proposed as the stochastic model. Thus, the solutions of (2.2) are stochastic processes, $L(t)$ and $C(t)$, giving the BOD and DO concentrations, respectively, at each distance $t$ downstream from the pollution source.

Assuming that $L_{a}, D_{B}, L_{0}$ and $C_{0}$ are random variables with finite second moments, the solution of the system (2.2) is given in [7]. According to Soong [8], the vector-valued stochastic process $\mathbf{X}(t), t \geqq 0$, is a mean square solution of $(2.2)$ if $\mathbf{X}(t)$ is mean square continuous on $[0, \infty)$, that is, $\mathbf{X}(t+h) \rightarrow \mathbf{m} \rightarrow \mathbf{X}(t)$ as $h \rightarrow 0$ for each $t \geqq 0 ; \mathbf{X}(0)=\mathbf{X}_{0}$ with probability one; and $\mathbf{A X}(t)+\mathbf{Y}$ is the mean square derivative of $\mathbf{X}(t)$ on $[0, \infty)$. Using the techniques in Soong [8, Chap. 7], the mean square solution of (2.2) may be represented as

$$
\mathbf{X}(t)=\boldsymbol{\Phi}(t) \mathbf{X}_{0}+\int_{0}^{t} \boldsymbol{\Phi}(t-s) \mathbf{Y} d s, \quad t \geqq 0,
$$


where

$$
\boldsymbol{\Phi}(t)=\left(\begin{array}{cc}
\exp \left[-\left(k_{1}+k_{3}\right) t / u\right] & 0 \\
-\frac{k_{1}}{k_{1}-k_{2}+k_{3}}\left[\exp \left(-k_{2} t / u\right)-\exp \left(-\left(k_{1}+k_{3}\right) t / u\right)\right] & \exp \left(-k_{2} t / u\right)
\end{array}\right)
$$

and the integral is a mean square integral. Thus, the mean square solution may be written as

$$
\left(\begin{array}{l}
L(t) \\
C(t)
\end{array}\right)=\left(\begin{array}{c}
\alpha_{1}(t) \\
\alpha_{2}(t)
\end{array}\right)\left(\begin{array}{c}
0 \\
\alpha_{3}(t)
\end{array}\right)\left(\begin{array}{l}
L_{0} \\
C_{0}
\end{array}\right)+\left(\begin{array}{c}
\beta_{1}\left(L_{a}, D_{B} ; t\right) \\
\beta_{2}\left(L_{a}, D_{B} ; t\right)
\end{array}\right)
$$

where $t \geqq 0$,

$$
\begin{aligned}
& \alpha_{1}(t)=\exp \left[-g_{1}(t)\right], \\
& \alpha_{2}(t)=\frac{k_{1}}{k_{1}-k_{2}+k_{3}}\left[\exp \left(-g_{1}(t)\right)-\exp \left(-g_{2}(t)\right)\right], \\
& \alpha_{3}(t)=\exp \left[-g_{2}(t)\right], \\
& \beta_{1}\left(L_{a}, D_{B} ; t\right)=\frac{L_{a}}{k_{1}+k_{3}}\left[1-\alpha_{1}(t)\right], \\
& \beta_{2}\left(L_{a}, D_{B} ; t\right)=\frac{k_{1} L_{a}}{k_{1}-k_{2}+k_{3}}\left[\frac{1-\alpha_{1}(t)}{k_{1}+k_{3}}-\frac{1-\alpha_{3}(t)}{k_{2}}\right] \\
& \quad+\frac{\left(k_{2} c_{S}-D_{B}\right)}{k_{2}}\left[1-\alpha_{3}(t)\right],
\end{aligned}
$$

and $g_{1}(t)=\left(k_{1}+k_{3}\right) t / u$ and $g_{2}(t)=k_{2} t / u$.

Thus, under various assumptions about the distributions of the random variables $L_{a}, D_{B}, L_{0}$ and $C_{0}$, simulated trajectories of the BOD and DO processes given by (2.3) may be obtained. In addition, as the results of the next section show, it is possible to obtain the joint distribution of $L(t)$ and $C(t)$ at each value of $t \geqq 0$ so that the statistical properties of the BOD and DO processes may be more completely determined.

3. Probability distribution of the mean square solution. The random vector differential equation (2.2) is of the form

$$
\dot{\mathbf{X}}(t)=\mathbf{h}(\mathbf{X}(t), \mathbf{B} ; t)
$$

with random initial conditions

$$
\mathbf{C}=\mathbf{X}(0),
$$

where $\mathbf{B}$ denotes a random vector. Let the joint probability density function of $\mathbf{C}$ and $\mathbf{B}$ be $f_{0}(\mathbf{c}, \mathbf{b})$. The random solution of (3.1) has the form

$$
\mathbf{X}(t)=\mathbf{g}(\mathbf{C}, \mathbf{B} ; t),
$$

where $\mathbf{h}$ and $\mathbf{g}$ are $\boldsymbol{n}$-vector-valued functions. 
We shall make use of the fundamental Liouville's theorem in the theory of dynamic systems to obtain the joint probability distribution of $\mathbf{X}(t)$. A proof of this theorem from a probabilistic point of view is due to Kozin [3].

THEOREM 3.1. Assume that a mean square solution of system (3.1) exists. Then the joint probability density function of $\mathbf{X}(t)$ and $\mathbf{B}, f(\mathbf{x}, \mathbf{b} ; t)$, satisfies the Liouville equation

$$
\frac{\partial f(\mathbf{x}, \mathbf{b} ; t)}{\partial t}+\sum_{j=1}^{n} \frac{\partial\left[f h_{j}\right]}{\partial x_{j}}=0,
$$

where $h_{j}$ and $x_{j}$ are the jth components of $\mathbf{h}$ and $\mathbf{x}$.

Thus, the problem of determining the joint probability density function $f(\mathbf{x}, \mathbf{b} ; t)$ using the Liouville equation (3.2) is an initial value problem for firstorder partial differential equations, the initial value being the joint probability density function of $\mathbf{C}$ and $\mathbf{B}, f_{0}(\mathbf{c}, \mathbf{b})$. As developed in Soong [8], the solution (that is, the joint probability density function of $\mathbf{X}(t)$ and $\mathbf{B})$ is given by

$$
f(\mathbf{x}, \mathbf{b} ; t)=\left.f_{0}(\mathbf{c}, \mathbf{b}) \exp \left\{-\int_{0}^{t} \nabla \cdot \mathbf{h}[\mathbf{x}=\mathbf{g}(\mathbf{c}, \mathbf{b} ; t), \mathbf{b} ; \tau] d \tau\right\}\right|_{\mathbf{c}=\mathbf{g}^{-1}(\mathbf{x}, \mathbf{b} ; t)}
$$

where $\nabla \cdot \mathbf{h}$ is the divergence of $\mathbf{h}$. Then the joint density function of $\mathbf{X}(t)$ can be found by integrating over $\mathbf{B}$,

$$
f(\mathbf{x} ; t)=\int_{R^{2}} f(\mathbf{x}, \mathbf{b} ; t) d \mathbf{b} .
$$

Now, we apply the above theorem to the random vector differential equation (2.2). Then

$$
\begin{gathered}
\mathbf{h}(\mathbf{X}(t), \mathbf{B} ; t)=\left(\begin{array}{l}
-\left(k_{1}+k_{3}\right) L(t) / u+L_{a} / u \\
-k_{1} L(t) / u-k_{2} C(t) / u+\left(k_{2} c_{S}-D_{B}\right) / u
\end{array}\right), \\
\mathbf{C}=\left(\begin{array}{l}
L_{0} \\
C_{0}
\end{array}\right) \text { and } \quad \mathbf{B}=\left(\begin{array}{l}
L_{a} \\
D_{B}
\end{array}\right) .
\end{gathered}
$$

Also,

$$
\begin{aligned}
\nabla \cdot \mathbf{h}=\left(\frac{\partial}{\partial L}, \frac{\partial}{\partial C}\right) \mathbf{h} & =-\frac{k_{1}+k_{3}}{u}-\frac{k_{2}}{u} \\
& =-\frac{1}{u}\left(k_{1}+k_{2}+k_{3}\right) .
\end{aligned}
$$

The mean square solution of (2.2) is given by (2.3) and is

$$
\begin{aligned}
\mathbf{g}(\mathbf{c}, \mathbf{b} ; t) & =\left(\begin{array}{cc}
\alpha_{1}(t) & 0 \\
\alpha_{2}(t) & \alpha_{3}(t)
\end{array}\right)\left(\begin{array}{l}
L_{0} \\
C_{0}
\end{array}\right)+\left(\begin{array}{l}
\beta_{1}\left(L_{a}, D_{B} ; t\right) \\
\beta_{2}\left(L_{a}, D_{B} ; t\right)
\end{array}\right) \\
& =\mathbf{X}(t) .
\end{aligned}
$$


If we let

$$
\mathbf{M}=\left(\begin{array}{cc}
\alpha_{1}(t) & 0 \\
\alpha_{2}(t) & \alpha_{3}(t)
\end{array}\right)
$$

(3.4) becomes

$$
\mathbf{X}(t)=\mathbf{M C}+\left(\begin{array}{l}
\beta_{1}\left(L_{a}, D_{B} ; t\right) \\
\beta_{2}\left(L_{a}, D_{B} ; t\right)
\end{array}\right)
$$

or

$$
\mathbf{M C}=\left(\begin{array}{l}
L(t)-\beta_{1}\left(L_{a}, D_{B} ; t\right) \\
C(t)-\beta_{2}\left(L_{a}, D_{B} ; t\right)
\end{array}\right)
$$

Then

$$
\begin{aligned}
\mathbf{C} & =\mathbf{M}^{-1}\left(\begin{array}{l}
L(t)-\beta_{1}\left(L_{a}, D_{B} ; t\right) \\
C(t)-\beta_{2}\left(L_{a}, D_{B} ; t\right)
\end{array}\right) \\
& =\frac{1}{\alpha_{1}(t) \alpha_{3}(t)}\left(\begin{array}{cc}
\alpha_{3}(t) & 0 \\
-\alpha_{2}(t) & \alpha_{1}(t)
\end{array}\right)\left(\begin{array}{l}
L(t)-\beta_{1}\left(L_{a}, D_{B} ; t\right) \\
C(t)-\beta_{2}\left(L_{a}, D_{B} ; t\right)
\end{array}\right) \\
& =\left(\begin{array}{c}
\frac{L-\beta_{1}}{\alpha_{1}} \\
-\frac{\alpha_{2}\left(L-\beta_{1}\right)+\alpha_{1}\left(C-\beta_{2}\right)}{\alpha_{1} \alpha_{3}}
\end{array}\right) \\
& =\mathbf{g}^{-1}(\mathbf{X}(t), \mathbf{B} ; t),
\end{aligned}
$$

if we suppress the arguments. Therefore, from (3.3) the joint probability density function of $\left(L(t), C(t), L_{a}, D_{B}\right)$ is given by

$$
\begin{aligned}
f\left(l, c, l_{a}, d_{B} ; t\right) & =f_{0}\left(l_{0}, c_{0}, l_{a}, d_{b}\right) \exp \left[-\int_{0}^{t} \nabla \cdot \mathbf{h} d \tau\right] \\
= & f_{0}\left(l_{0}, c_{0}, l_{a}, d_{B}\right) \exp \left[-\int_{0}^{t}-\left(k_{1}+k_{2}+k_{3}\right) / u d \tau\right] \\
= & f_{0}\left(\frac{l-\beta_{1}}{\alpha_{1}}, \frac{-\alpha_{2}\left(l-\beta_{1}\right)+\alpha_{1}\left(c-\beta_{2}\right)}{\alpha_{1} \alpha_{3}}, l_{a}, d_{B}\right) \\
& \cdot \exp \left[\left(k_{1}+k_{2}+k_{3}\right) t / u\right]
\end{aligned}
$$

since $\mathbf{c}=\mathbf{g}^{-1}(\mathbf{X}, \mathbf{B} ; t)=\left(L_{0}, C_{0}\right)^{T}$. Then the joint density function of $\mathbf{X}(t)=$ $(L(t), C(t))^{T}$ is obtained from (3.5) by integration:

$$
f_{1}(l, c ; t)=\int_{-\infty}^{\infty} \int_{-\infty}^{\infty} f\left(l, c, l_{a}, d_{B} ; t\right) d l_{a} d d_{B} .
$$

The moments of $L(t)$ and $C(t)$ at each $t \geqq 0$ may be evaluated using (3.6).

To illustrate these results, in the next section we shall consider several distributions for $L_{0}, C_{0}, L_{a}$ and $D_{B}$ and obtain the joint probability density 
functions of the BOD and DO processes. In addition, we shall present simulated trajectories of $L(t)$ and $C(t)$ obtained from the mean square solutions and give the mean functions and variance functions obtained by numerical integration from the probability density function (3.6).

4. Examples. The data and the probability distributions of $L_{0}, C_{0}, L_{a}$ and $D_{B}$ which are used in the examples given in this section are consistent with the values that were used by Thayer and Krutchkoff [10] taken from a study of the Sacramento River. For comparison, the solutions of the deterministic differential equations (2.1) have been plotted in Fig. 1 using the values $k_{1}=0.35, k_{2}=0.75$, $k_{3}=0.20, l_{0}=6.8, c_{0}=8.7(\mathrm{ppm}), l_{a}=0.2, d_{B}=0.1, c_{S}=10$, and $u=7.5$ (miles per day) similar to those used in [10].

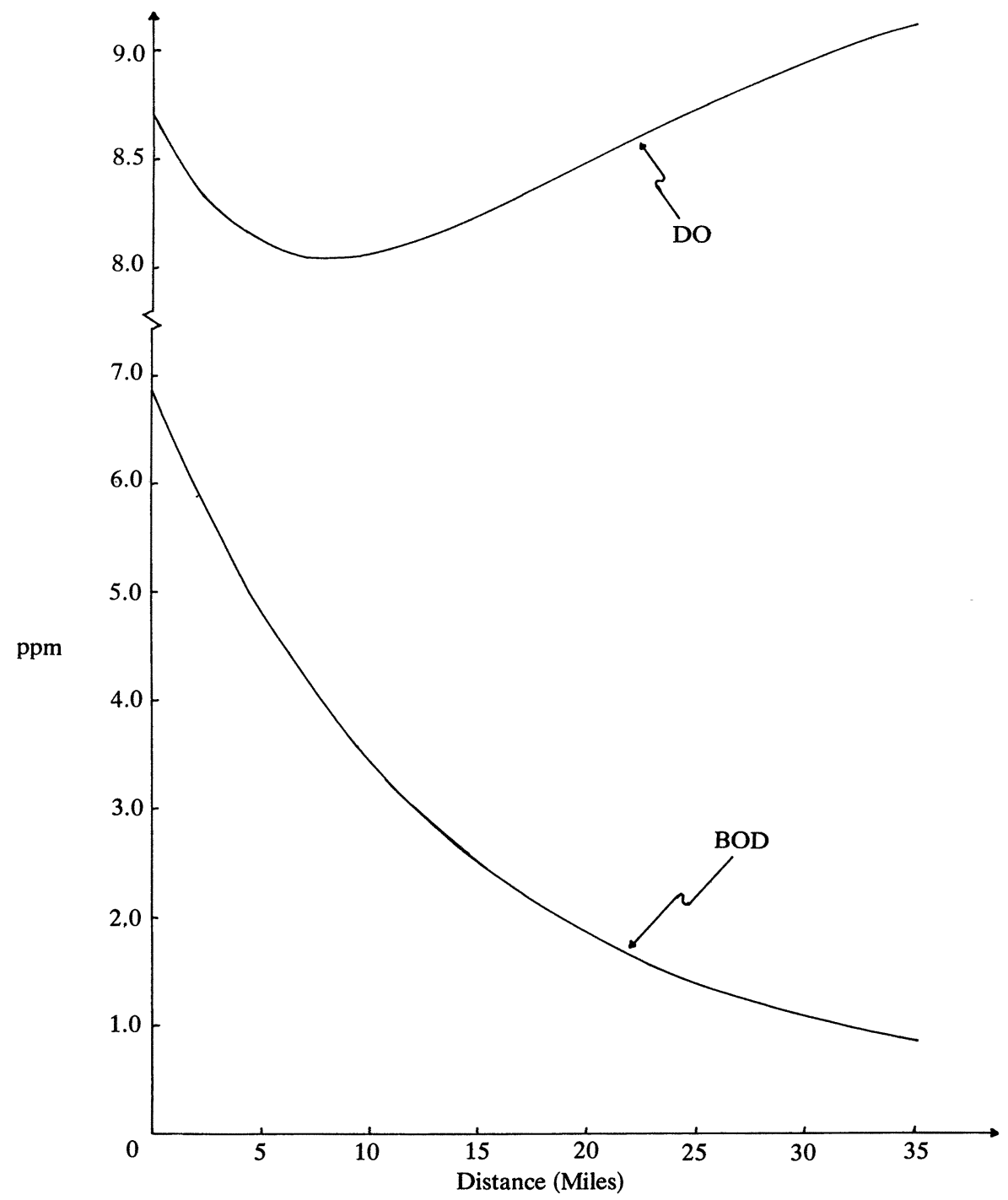

Fig. 1. Deterministic case 
We now assume various distributions for the random variables $L_{0}, C_{0}, L_{a}$ and $D_{B}$ and obtain the joint density of $L(t)$ and $C(t)$ for each $t \geqq 0$.

Case 1. Suppose $L_{0}, C_{0}, L_{a}$ and $D_{B}$ are independent random variables, $L_{a}$ and $D_{B}$ are uniformly distributed on $(0,0: 4)$ and $(0,0.2)$, respectively, and $L_{0}$ and $C_{0}$ have truncated normal distributions with respective means 6.8 and 8.7 (ppm) and variances 1.0 and 0.03 . The values of $k_{1}, k_{2}, k_{3}, c_{s}$ and $u$ will be $0.35,0.75$, $0.20,10$ and 7.5 , respectively, as in the deterministic case. Then the joint probability density function of $L_{0}, C_{0}, L_{a}$ and $D_{B}$ is given by

$$
f_{0}\left(l_{0}, c_{0}, l_{a}, d_{B}\right)=\left\{\begin{array}{rr}
\frac{d_{1} d_{2}}{2 \pi \sqrt{0.03}(0.08)} \exp \left[-\frac{1}{2}\left(l_{0}-6.8\right)^{2}-\frac{\left(c_{0}-8.7\right)^{2}}{0.06}\right], \\
0<l_{a}<0.4, \quad 0<d_{B}<0.2, \\
0 \leqq l_{0}<\infty, \quad 0 \leqq c_{0} \leqq c_{S}, \\
0, \text { otherwise, }
\end{array}\right.
$$

where $d_{1}$ and $d_{2}$ are truncation factors for the densities of $L_{0}$ and $C_{0}$, respectively. From equations (3.5) and (4.1) we obtain the joint probability density function of $\mathbf{X}(t)$ and $\mathbf{B}$,

$$
\begin{aligned}
& f(\mathbf{x}, \mathbf{b} ; t)= \exp \left[\left(k_{1}+k_{2}+k_{3}\right) \frac{t}{u}\right] \frac{d_{1} d_{2}}{2 \pi \sqrt{0.03}(0.08)} \\
&(4.2) \quad \cdot \exp \left\{-\frac{1}{2}\left[\frac{\left(l-\beta_{1}\right)}{\alpha_{1}}-6.8\right]^{2}-\frac{1}{2(0.03)}\left[\frac{-\alpha_{2}\left(l-\beta_{1}\right)+\alpha_{1}\left(c-\beta_{2}\right)}{\alpha_{1} \alpha_{3}}-8.7\right]^{2}\right\} \\
& \text { if } \beta_{1} \leqq l<\infty, \quad c_{*} \leqq c \leqq c^{*}, \quad 0<l_{a}<0.4, \quad 0<d_{B}<0.2,
\end{aligned}
$$

where $\quad c_{*}=\max \left\{0,\left(\alpha_{2} / \alpha_{1}\right)\left(l-\beta_{1}\right)+\beta_{2}\right\}, \quad c^{*}=\min \left\{c_{S}, c_{S} \alpha_{3}+\left(\alpha_{2} / \alpha_{1}\right)\left(l-\beta_{1}\right)\right.$ $\left.+\beta_{2}\right\}$, and

$$
f(\mathbf{x}, \mathbf{b} ; t)=0, \quad \text { otherwise }
$$

The joint density function of $L(t)$ and $C(t)$ can then be found from (4.2) by integrating over $l_{a}$ and $d_{B}$. Then the mean functions, $E[L(t)]$ and $E[C(t)]$, and the variance functions, $\sigma_{L}^{2}(t)$ and $\sigma_{C}^{2}(t)$, may be obtained by the usual integration of the joint density function of $L(t)$ and $C(t)$. Figs. 2(a) and 2(b) show simulated trajectories of the solution processes $L(t)$ and $C(t)$ using the assumed distributions of $L_{0}, C_{0}, L_{a}$ and $D_{B}$ above and the equations (2.3). Also, the mean functions and variance functions were computed numerically (using Gauss quadrature formulas for the numerical integration), and the results are shown in Figure 2(c).

Case 2. We now assume that $L_{0}$ and $C_{0}$ have a truncated bivariate normal distribution with correlation coefficient $\rho$. Under the assumptions (iv) and (v) in $\S 1$, it is realistic to assume that $L_{a}$ and $D_{B}$ are independent and also that they are 


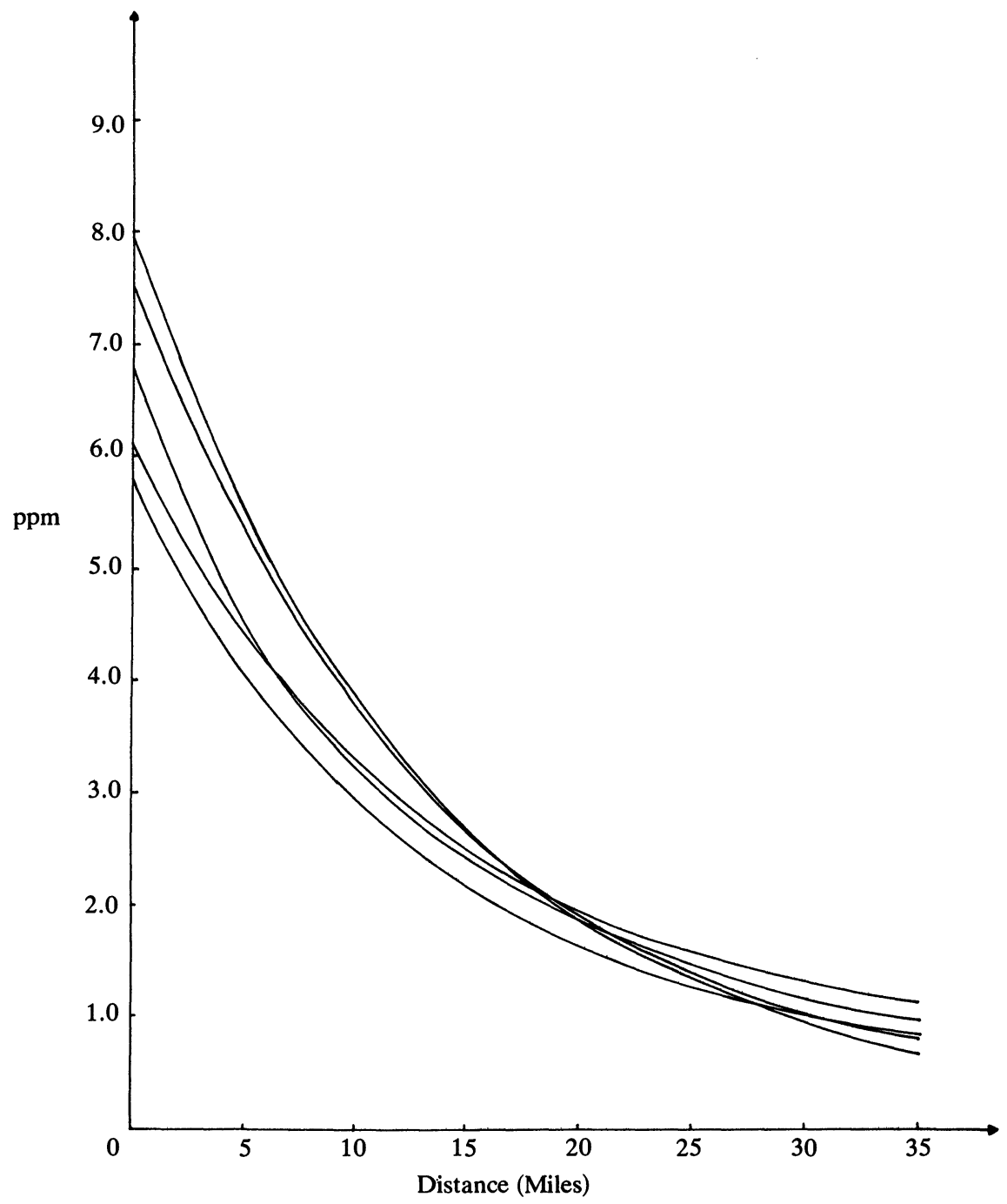

FIG. 2(a). Simulated trajectories of BOD for Case 1

independent of $\left(L_{0}, C_{0}\right)$. The joint probability density function of $\left(L_{0}, C_{0}\right)$ is given by

$$
\begin{array}{r}
g_{1}\left(l_{0}, c_{0}\right)=\frac{d_{3}}{2 \pi \sqrt{0.03} \sqrt{1-\rho^{2}}} \exp \left\{-\frac{1}{2\left(1-\rho^{2}\right)}\left[\left(l_{0}-6.8\right)^{2}\right.\right. \\
\left.\left.-\frac{2 \rho}{\sqrt{0.03}}\left(l_{0}-6.8\right)\left(c_{0}-8.7\right)+\frac{\left(c_{0}-8.7\right)^{2}}{0.03}\right]\right\} \\
\quad \text { if } 0 \leqq l_{0}<\infty, \quad 0 \leqq c_{0} \leqq c_{S},
\end{array}
$$




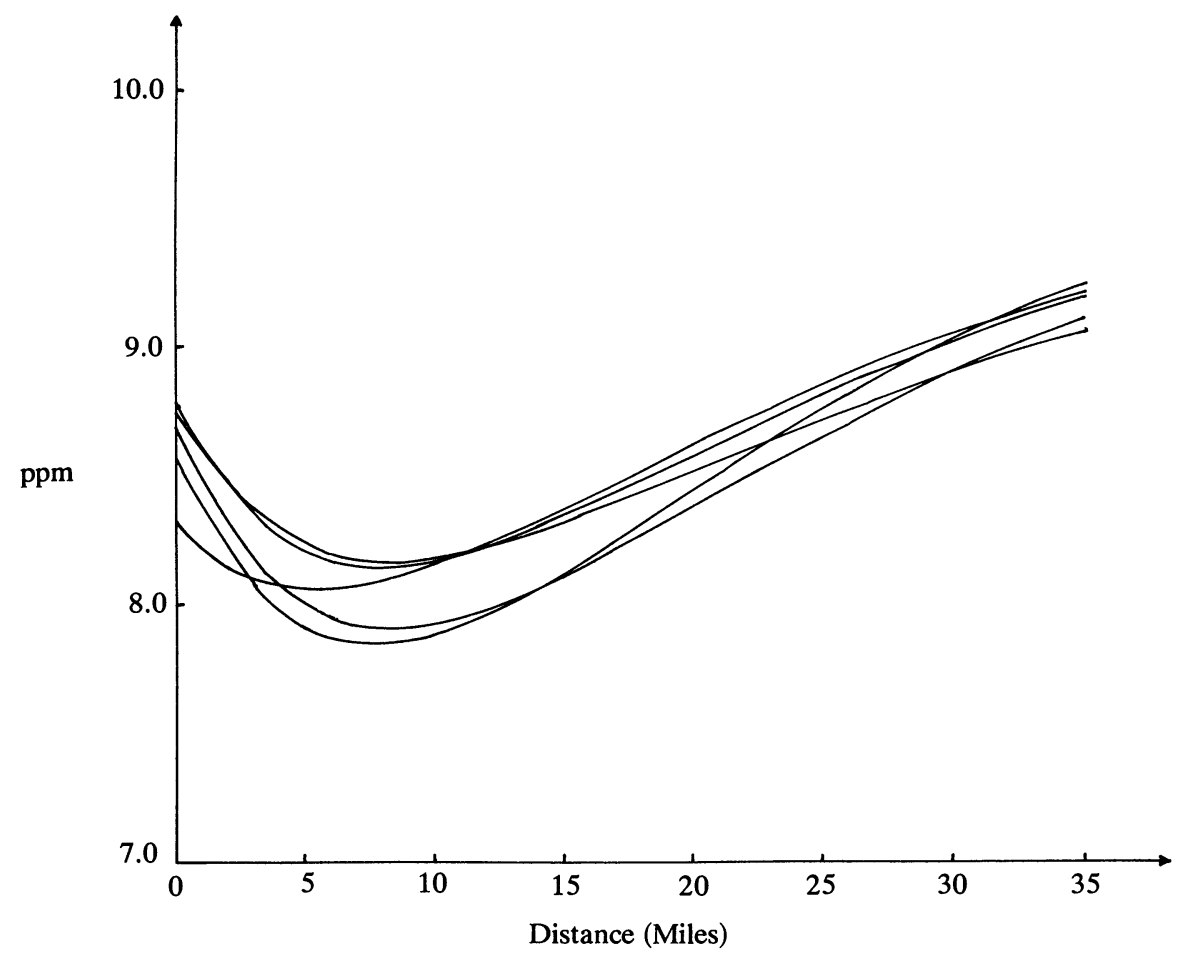

FIG. 2(b). Simulated trajectories of DO for Case 1

and is zero otherwise, where $d_{3}$ is the truncation factor. Thus, the joint density of $\left(L_{0}, C_{0}, L_{a}, D_{B}\right)$ is

$$
g_{0}\left(l_{0}, c_{0}, l_{a}, d_{B}\right)=\frac{1}{0.08} g_{1}\left(l_{0}, c_{0}\right), \quad 0 \leqq l_{a} \leqq 0.4, \quad 0 \leqq d_{B} \leqq 0.2 .
$$

Thus, from the results of $\S 3$ and (4.3), the joint density function of $\mathbf{X}(t)$ and $\mathbf{B}$ is given by

$$
\begin{array}{r}
g(\mathbf{x}, \mathbf{b} ; t)=\frac{d_{3}}{(0.08) 2 \pi \sqrt{0.03} \sqrt{1-\rho^{2}}} \exp \left\{-\frac{1}{2\left(1-\rho^{2}\right)}\left[\left(\frac{l-\beta_{1}}{\alpha_{1}}-6.8\right)^{2}\right.\right. \\
-\frac{2 \rho}{\sqrt{0.03}}\left(\frac{l-\beta_{1}}{\alpha_{1}}-6.8\right)\left(\frac{\alpha_{2}\left(\beta_{1}-l\right)+\alpha_{1}\left(c-\beta_{2}\right)}{\alpha_{1} \alpha_{3}}-8.7\right) \\
\left.\left.+\left(\frac{\alpha_{2}\left(\beta_{1}-l\right)+\alpha_{1}\left(c-\beta_{2}\right)}{\alpha_{1} \alpha_{3}}-8.7\right)^{2} / 0.03\right]\right\} \\
\cdot \exp \left[\left(k_{1}+k_{2}+k_{3}\right) t / u\right], 0 \leqq l_{a} \leqq 0.4, \quad 0 \leqq d_{B} \leqq 0.2, \\
\beta_{1} \leqq l<\infty, \quad c_{*} \leqq c \leqq c^{*},
\end{array}
$$

and $g(\mathbf{x}, \mathbf{b} ; t)=0$, otherwise. Hence, as before the joint density function of $L(t)$ and $C(t)$ at each $t \geqq 0$ may be obtained. Figs. 3(a)-(b) and 4(a)-(b) show simulated 


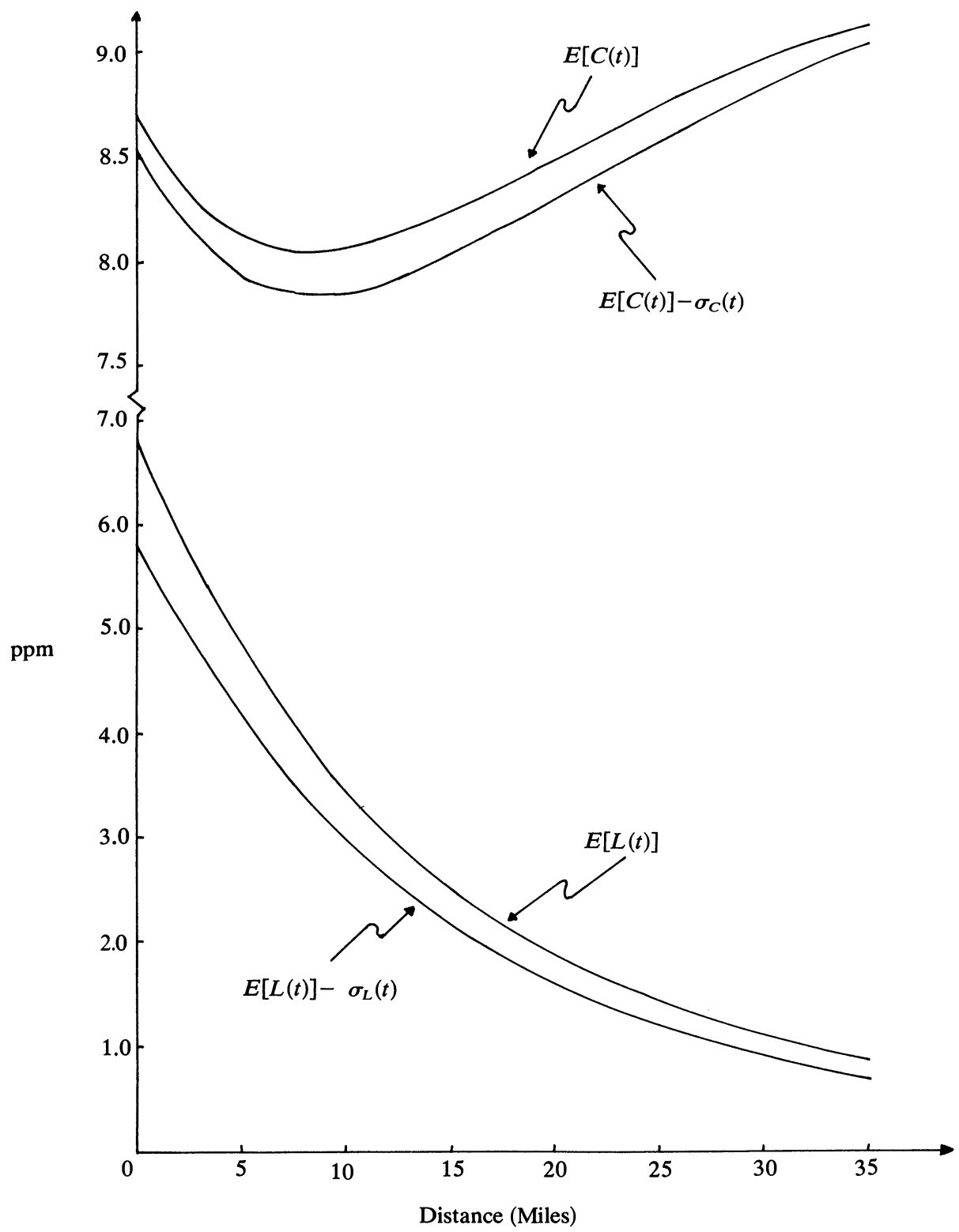

FIG. 2(c). Mean and standard deviation functions of BOD and DO for Case 1

trajectories of $L(t)$ and $C(t)$ obtained by generating random numbers $l_{a}, d_{B}$ and $\left(l_{0}, c_{0}\right)$ from the assumed distributions with $\rho= \pm 0.5$, respectively; and using the same values of $k_{1}, k_{2}, k_{3}, c_{s}$ and $u$ as were used in Case 1. Also, Figs. 3(c) and 4(c) show the mean and variance functions of $C(t)$ as computed by numerical integration from the joint density function (4.4) with $\rho=0.5$ and -0.5 , respectively. The mean and variance functions of $L(t)$ in this case are similar to those for Case 1 and hence are not plotted. 


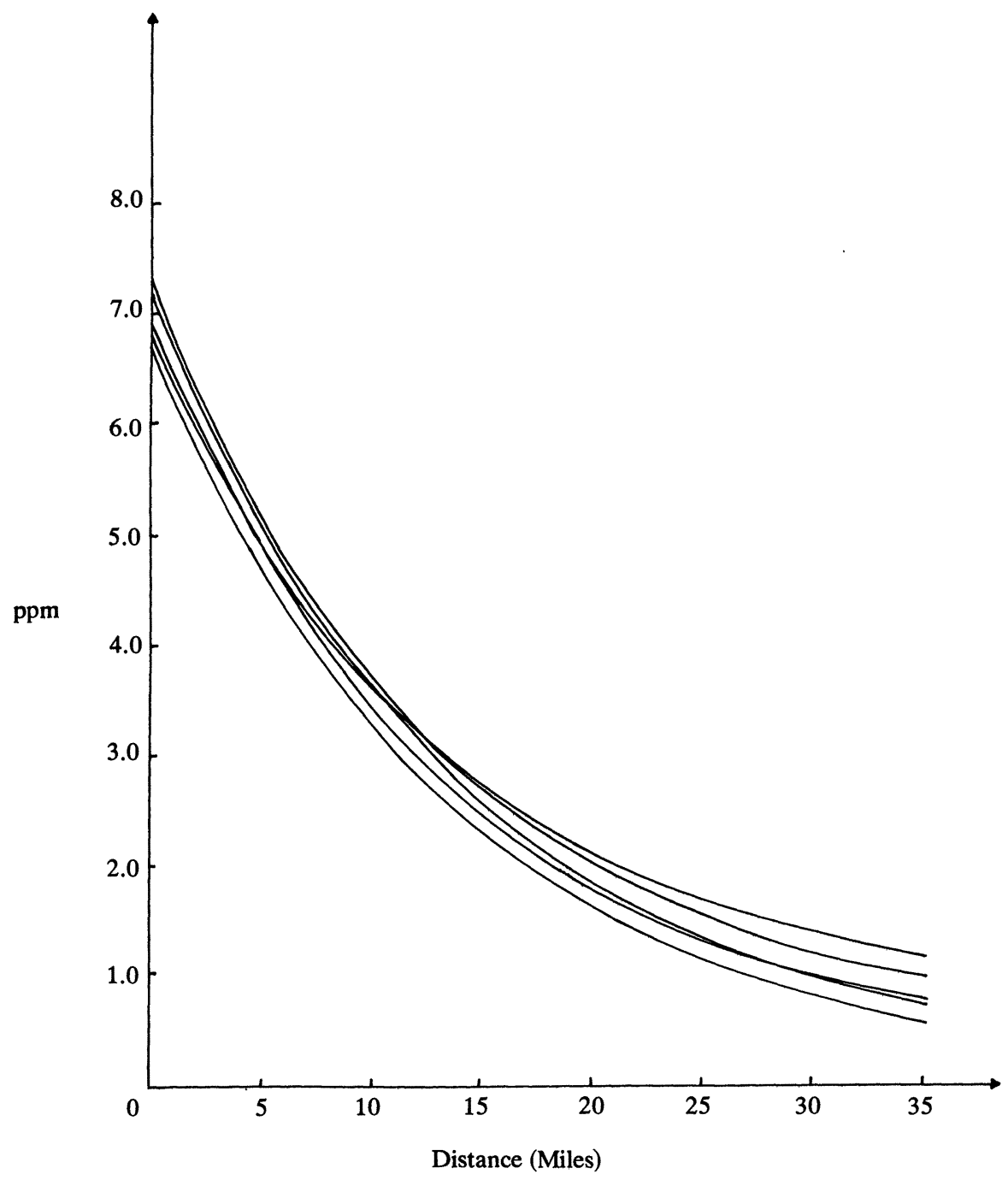

FIG. 3(a). Simulated trajectories of BOD for Case $2(\rho=0.5)$

We remark that if $\rho=1, L_{0}$ and $C_{0}$ are linearly related by $L_{0}=r C_{0}+s$, where $r=\sigma_{L_{0}} / \sigma_{C_{0}}=1 / \sqrt{0.03}$ and $s=6.8-\sqrt{0.03} \times(8.7)$. Hence, in this case, we need only the joint probability density function of $\left(C_{0}, L_{a}, D_{B}\right)$ which simplifies the joint density of $L(t)$ and $C(t)$.

Case 3. Finally, suppose the random vector $\mathbf{Y}=\left(L_{0}, C_{0}, L_{a}, D_{B}\right)$ has a truncated multivariate normal distribution with mean vector $\mu$ and covariance matrix $\mathrm{V}$. Then the joint density function of $\left(L_{0}, C_{0}, L_{a}, D_{B}\right)$ is

$g_{0}(\mathbf{y} ; \boldsymbol{\mu}, \mathbf{V})=\left\{\begin{array}{l}\frac{d_{4}|\mathbf{P}|^{1 / 2}}{(2 \pi)^{2}} \exp \left[-\frac{1}{2}(\mathbf{y}-\boldsymbol{\mu})^{T} \mathbf{P}(\mathbf{y}-\boldsymbol{\mu})\right], \quad 0 \leqq l_{0}, l_{a}, d_{B}<\infty, \quad 0 \leqq c_{0} \leqq c_{S}, \\ 0, \quad \text { otherwise, }\end{array}\right.$ 


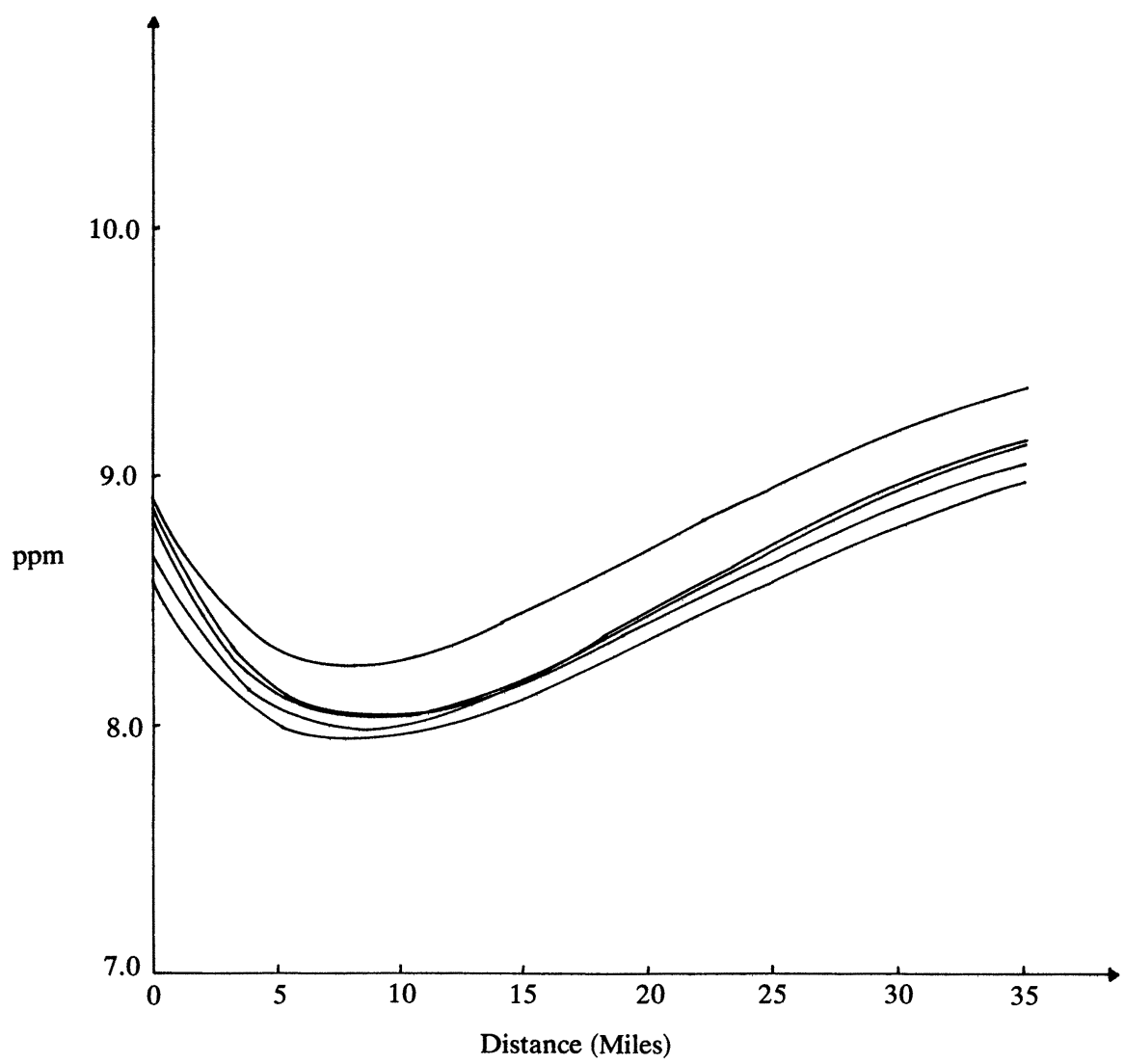

FIG. 3(b). Simulated trajectories of DO for Case $2(\rho=0.5)$

where $d_{4}$ is the truncation factor and $\mathbf{P}=\mathbf{V}^{-1}$. As before, it is easy to find the joint density function of $\mathbf{X}(t)$ and $\mathbf{B}$ from $g_{0}$,

$$
g(\mathbf{x}, \mathbf{b} ; t)=\exp \left[\left(k_{1}+k_{2}+k_{3}\right) \frac{t}{u}\right] \frac{d_{4}|\mathbf{P}|^{1 / 2}}{(2 \pi)^{2}} \exp \left[-\frac{1}{2}\left(\mathbf{y}^{\prime}-\boldsymbol{\mu}\right)^{T} \mathbf{P}\left(\mathbf{y}^{\prime}-\boldsymbol{\mu}\right)\right]
$$

where $y_{1}^{\prime}=\left(l-\beta_{1}\right) / \alpha_{1} \geqq 0,0 \leqq y_{2}^{\prime}=\left[\alpha_{2}\left(\beta_{1}-l\right)+\alpha_{1}\left(c-\beta_{2}\right)\right] / \alpha_{1} \alpha_{3} \leqq c_{S}, 0 \leqq y_{3}^{\prime}=$ $l_{a}<\infty$ and $0 \leqq y_{4}^{\prime}=d_{B}<\infty$ and $g(\mathbf{x}, \mathbf{b} ; t)=0$, otherwise.

5. Discussion. The results of $\S \S 3$ and 4 allow probability statements to be made concerning the value of DO at each distance $t$ downstream from a pollution source. Hence, the probability that, for given initial variables $L_{0}$ and $C_{0}$, the DO level will be below a specified amount can be computed. The minimum DO level is of great importance since the fish population and other aquatic life would be endangered if DO falls below a certain value for even a short period of time. 


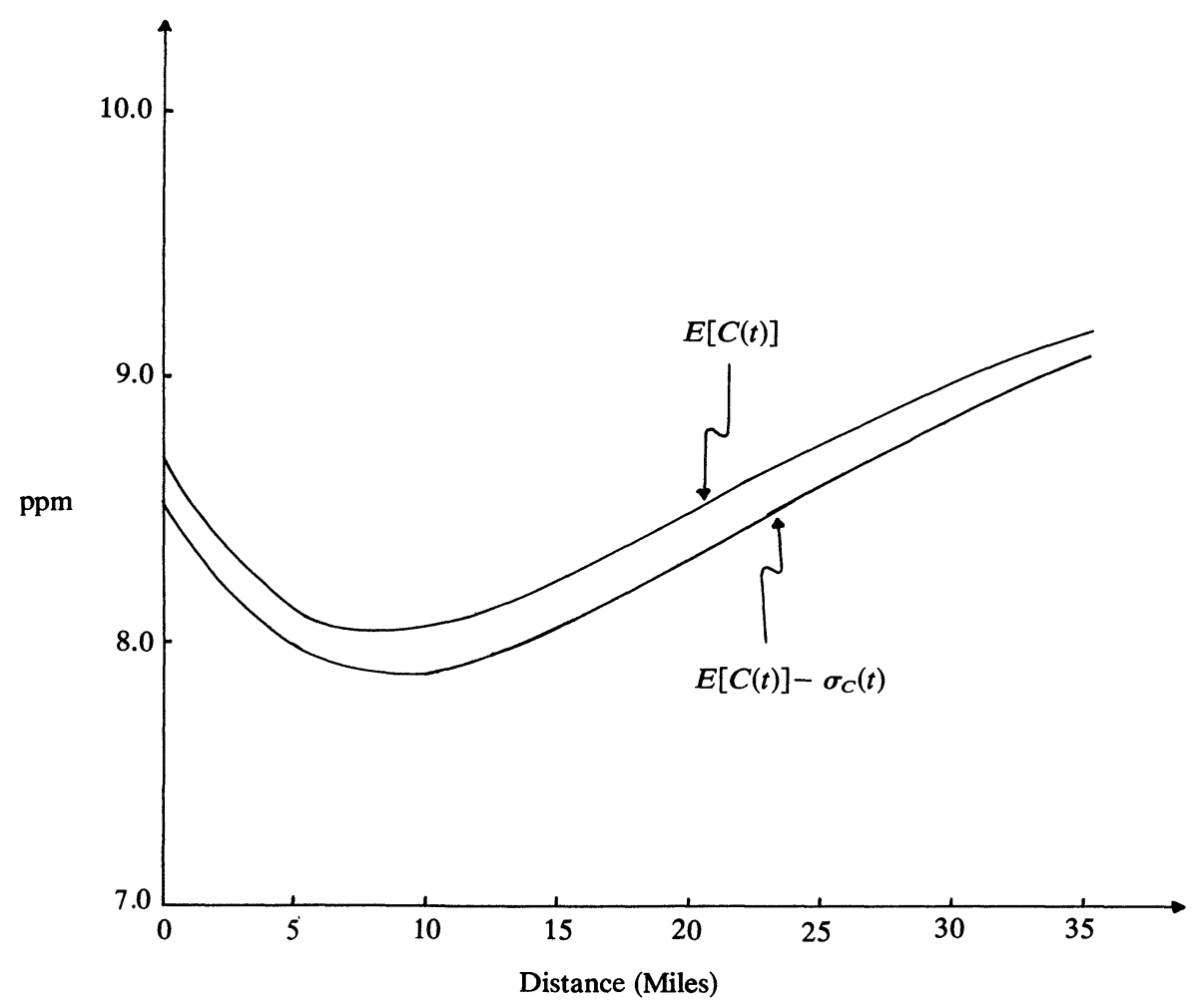

FIG. 3(c). Mean and standard deviation functions of DO $(\rho=0.5)$

The particular distributions used in $\S 4$ seem to be reasonable since DO and BOD can be measured at the source of pollution to yield at least an approximate probability distribution for $L_{0}$ and $C_{0}$. The normal distribution is a reasonable one even if no randomness other than measurement error is assumed. The uniform distribution on $L_{a}$ and $D_{B}$ may be regarded as simply placing values of $l_{a}$ and $d_{B}$ in an interval with ignorance of the exact probability distributions.

The mean function and variance function of DO shown in Fig. 2(c) for Case 1 support the contention of Thayer and Krutchkoff [10] that the variance of dissolved oxygen is greater in the "oxygen sag" region of the DO profile than in the other regions. Hence, our results agree with the experimental results of Thayer and Krutchkoff [10]. It is also interesting to note that if $L_{0}$ and $C_{0}$ have correlation coefficient $\rho=0.5$ the variance at each $t \geqq 0$ is smaller than for the uncorrelated case, whereas if $\rho=-0.5$ the variance at each $t$ is larger than it is for $\rho=0$. This is also evidenced in the simulated trajectories of BOD and DO shown for the three values of $\rho=0,0.5$ and -0.5 in Figs: 2(a)-(b), 3(a)-(b) and 4(a)-(b), respectively. Hence, it seems that if the correlation coefficient of $L_{0}$ and $C_{0}$ could be estimated efficiently, then it would yield valuable information concerning the behavior of $L(t)$ and $C(t)$ for $t>0$. It should also be noted that in Figures 2(c), 3(c) and $4(\mathrm{c})$ the mean functions coincide with the deterministic trajectories given in 


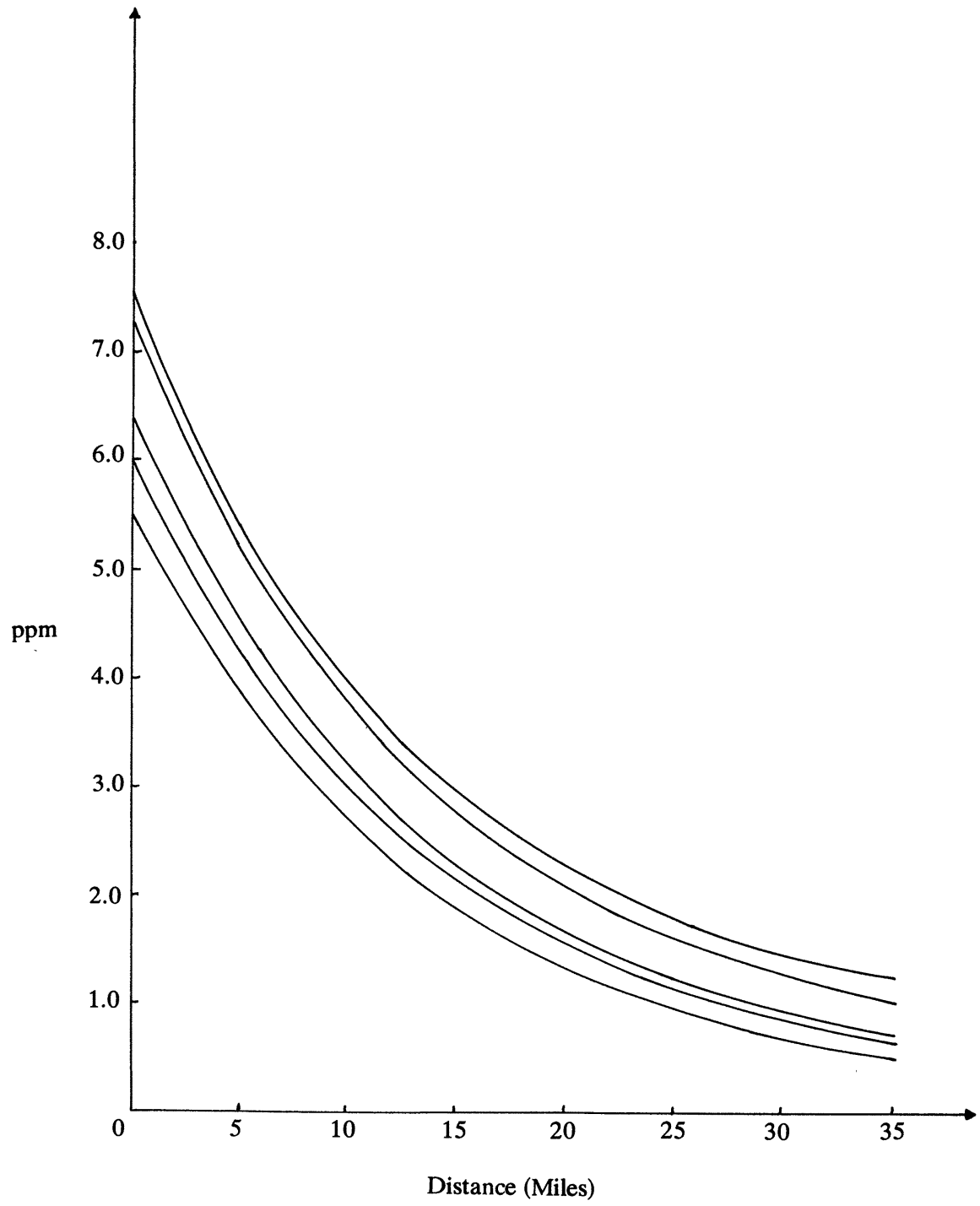

FIG. 4(a). Simulated trajectories of BOD for Case $2(\rho=-0.5)$

Fig. 1 since the solution of the random differential equation is linear in the random variables.

Obviously, other distributions for $L_{0}, C_{0}, L_{a}$ and $D_{B}$ may be handled as easily as those in $\S 4$. Also, with obvious modifications, the quantities $K_{1}, K_{2}$ and $K_{3}$ could be considered as random variables instead of (or in addition to) $L_{a}$ and $D_{B}$. The Liouville equation (3.2) may also be applied in this case.

Acknowledgment. The authors are grateful to the referees for their valuable suggestions. 


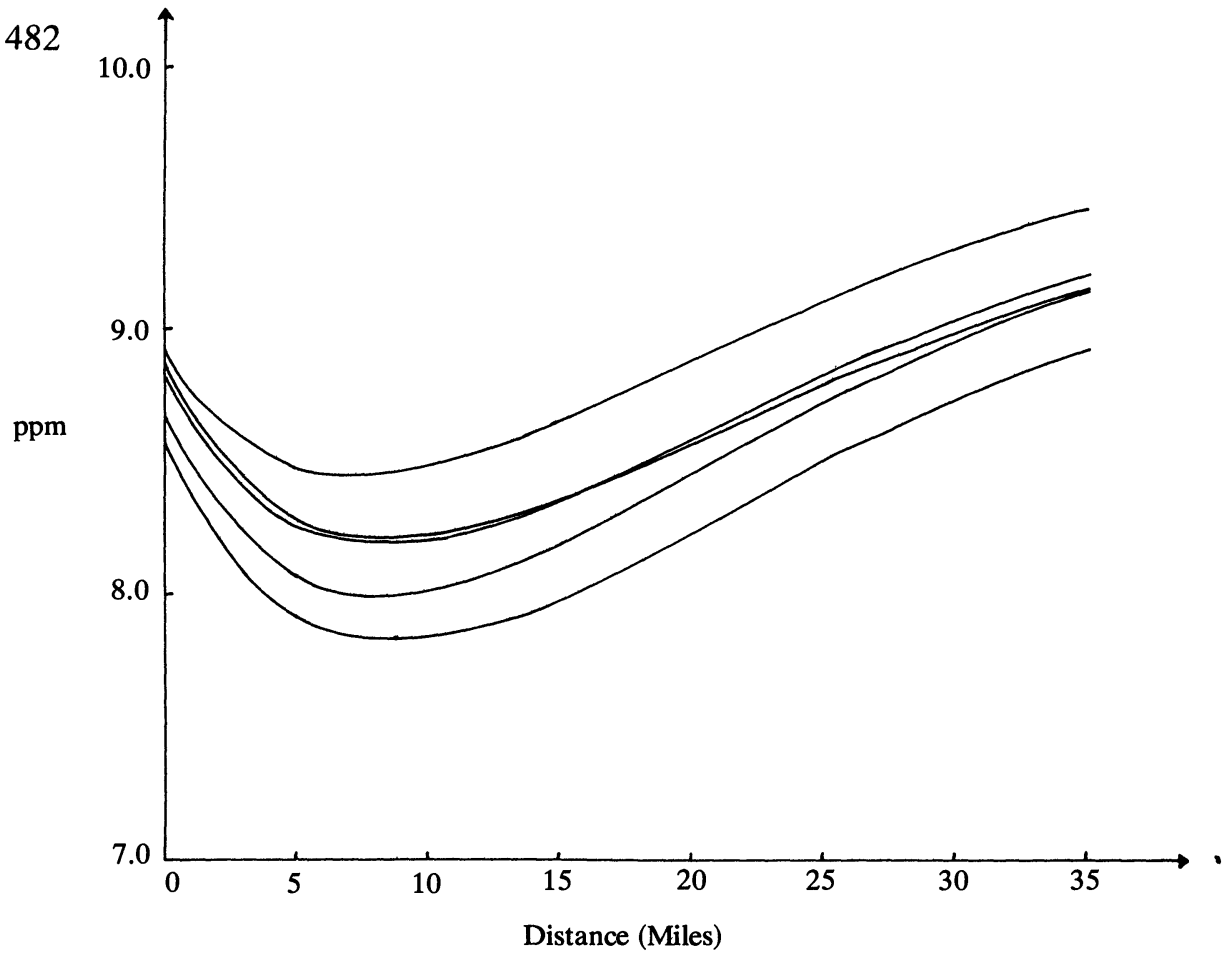

FIG. 4(b). Simulated trajectories of DO for Case $2(\rho=-0.5)$

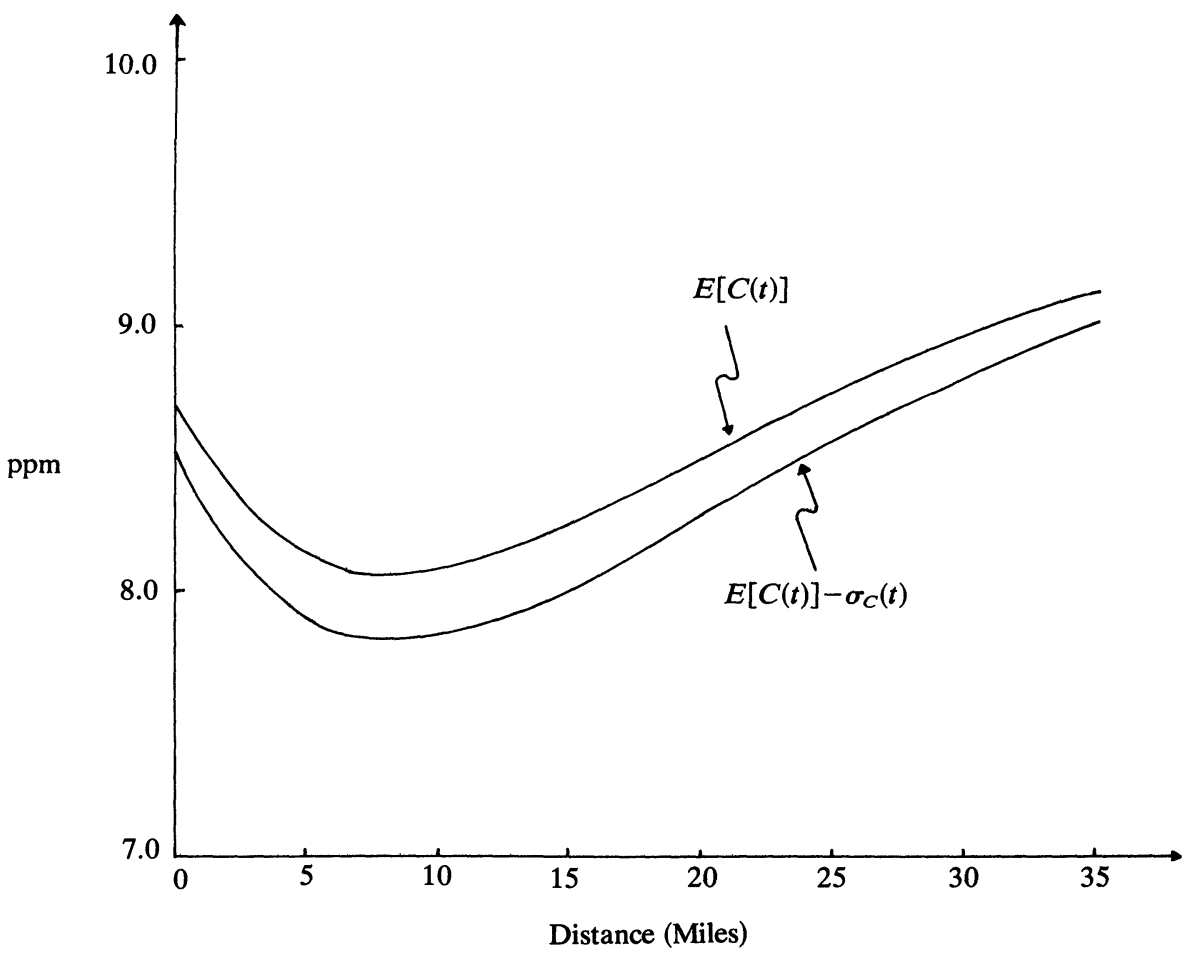

FIG. 4(c). Mean and standard deviation functions of DO for Case $2(\rho=-0.5)$ 


\section{REFERENCES}

[1] WiLliam E. DoBbins, BOD and oxygen relationships in streams, J. Sanitary Engineering Division, Amer. Soc. Civil Engrs., (SA3) 90 (1964), pp. 53-78.

[2] V. GOURISHANKAR AND R. L. LAWSON, Optimal control of water pollution in a river stream, Internat. J. Systems Sci., 6 (1975), pp. 201-216.

[3] F. KozIN, On the probability densities of the output of some random systems, J. Appl. Mech., 28 (1961), pp. 161-165.

[4] D. P. LOUCKS AND W. R. LYNN, Probabilistic models for predicting stream quality, Water Resources Res., 2 (1966), pp. 593-605.

[5] D. J. O'ConNor, Oxygen balance of an estuary, Trans. Amer. Soc. Civil Engrs., 126 (1961), 556.

[6] - The temporal and spatial distribution of dissolved oxygen in streams, Water Resources Res., 3 (1967), pp. 65-79.

[7] W.J. PADGETT, A stochastic model for stream pollution, Math. Biosci., 25 (1975), pp. 309-317.

[8] T. T. SoONG, Random Differential Equations in Science and Engineering, Academic Press, New York, 1973.

[9] H. W. STREeTER AND E. B. PHELPS, A study of the pollution and natural purification of the Ohio River, Public Health Bulletin 146, U.S. Public Health Service, Washington, D.C., 1925.

[10] RICHARD P. THAYER AND RICHARD G. KRUTCHKOFF, Stochastic model for BOD and DO in streams, J. Sanitary Engineering Division, Amer. Soc. Civil Engrs., (SA3), 93 (1967), pp. 59-72. 